\title{
Non-Fermi-Liquid Behavior in Transport Through Co-Doped Au Chains
}

\author{
S. Di Napoli, ${ }^{1}$ A. Weichselbaum, ${ }^{2}$ P. Roura-Bas, ${ }^{1}$ A. A. Aligia,${ }^{3}$ Y. Mokrousov, ${ }^{4}$ and S. Blügel ${ }^{4}$ \\ ${ }^{1}$ Departamento de Física de la Materia Condensada, CAC-CNEA, Avenida General Paz 1499, 1650 San Martín, Provincia de Buenos \\ Aires, Argentina and Consejo Nacional de Investigaciones Científicas y Técnicas, CONICET, Buenos Aires C1033AAJ, Argentina \\ ${ }^{2}$ Ludwig Maximilians University and Arnold Sommerfeld Center for Theoretical Physics, 80333 Munich, Germany \\ ${ }^{3}$ Centro Atómico Bariloche and Instituto Balseiro, Comisión Nacional de Energía Atómica, 8400 Bariloche, Argentina \\ ${ }^{4}$ Peter Grünberg Institut and Institute for Advanced Simulation, Forschungszentrum Jülich and JARA, 52425 Jülich, Germany
}

(Received 12 October 2012; published 6 May 2013)

\begin{abstract}
We calculate the conductance as a function of temperature $G(T)$ through Au monatomic chains containing one Co atom as a magnetic impurity, and connected to two conducting leads with a fourfold symmetry axis. Using the information derived from $a b$ initio calculations, we construct an effective model $\hat{H}_{\text {eff }}$ that hybridizes a $3 d^{7}$ quadruplet at the Co site with two $3 d^{8}$ triplets through the hopping of $5 d_{x z}$ and $5 d_{y z}$ electrons of Au. The quadruplet is split by spin anisotropy due to spin-orbit coupling. Solving $\hat{H}_{\text {eff }}$ with the numerical renormalization group we find that at low temperatures $G(T)=a-b \sqrt{T}$ and the ground state impurity entropy is $\ln (2) / 2$, a behavior similar to the two-channel Kondo model. Stretching the chain leads to a non-Kondo phase, with the physics of the underscreened Kondo model at the quantum critical point.
\end{abstract}

DOI: 10.1103/PhysRevLett.110.196402

PACS numbers: 71.10.Hf, 73.23.- b, 75.20.Hr

Transport properties in nanoscopic systems are being extensively studied due to potential applications and their role in basic research. In particular, the Kondo effect, in which the spin $1 / 2$ of a molecule or a quantum dot (QD) is screened by one channel of conduction electrons below a characteristic temperature $T_{K}$, has been experimentally observed and the conductance as a function of temperature $G(T)$ is in excellent agreement with theory $[1,2]$. In spite of the large effect of correlations, these systems are Fermi liquids where, for example, the conductance has the expected behavior $G(T) \simeq G(0)-a T^{2}$ for $T \ll T_{K}$, with $T_{K}$ the Kondo temperature and $a$ some constant. More recently, the underscreened Kondo (UK) effect, leading to singular Fermi liquid physics [3,4] has been observed, and quantum phase transitions (QPTs) involving partially Kondo screened spin-1 molecular states were induced by externally controlled parameters [5-7].

The overscreened Kondo effect, the simplest manifestation of which is the two-channel Kondo (2CK) model, is even more interesting because inelastic scattering persists even at vanishing temperatures and excitation energies. Therefore, the system is a non-Fermi liquid, exhibiting fascinating low-energy properties [8-10]. In particular, the impurity contribution to the entropy is $\ln (2) / 2$ and the conductance per channel at low $T$ has the form $G(T) \simeq$ $G_{0} / 2 \pm a \sqrt{T}$, where $G_{0}$ is the conductance at zero temperature in the one-channel case. However, experimental observations of $2 \mathrm{CK}$ physics have been elusive, particularly due to its instability against the asymmetry of the channels [10]. For example, while 2CK physics is expected to take place at the QPT in the model of two spin-1/2 Kondo impurities $[9,10]$, interchannel charge transfer spoils the quantum critical point (QCP) [10] and its observation in double QD systems [11].

Oreg and Goldhaber-Gordon [12] proposed to modify the simplest setup of the one-electron transistor (a QD between two conducting leads) by adding a second large QD with level spacing $\delta<T_{K}$, which acts as a second channel of conduction electrons, with gate voltage finetuned towards the QCP. Following this proposal, the differential conductance as a function of bias voltage $V_{b}$ was measured and a $\sqrt{V_{b}}$ behavior, characteristic of $2 \mathrm{CK}$ physics was observed for $e V_{b}>k_{B} T$ [13].

In this Letter, we show that $2 \mathrm{CK}$ physics is expected in transport through Co doped Au chains connected to conducting leads with a fourfold axis, as shown in Fig. 1(b). In this case, the mobile electrons of the two channels are the

(a) CHAIN

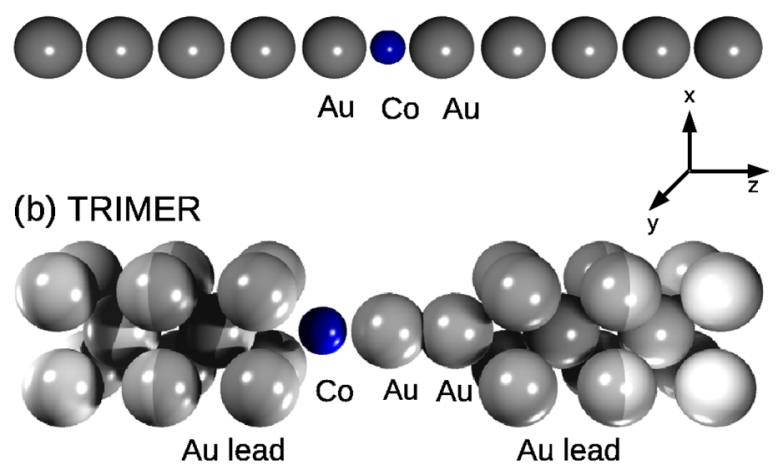

FIG. 1 (color online). Structure of the systems studied. (a) Au chain doped with $\mathrm{Co}$; (b) trimer with one Co atom connected to body-centered-cubic leads. The shaded region indicates the unit cell used. 
$5 d x z$ and $y z$ electrons of Au. An advantage over the proposal of Ref. [12] is that both channels are related by SU(2) symmetry in the effective model $\hat{H}_{\text {eff }}$ and therefore fine-tuning is not necessary. Furthermore, by stretching the chain, it appears possible to pass through a QCP with underscreened Kondo physics to another phase with reduced conductance, without Kondo behavior. To the best of our knowledge, our model has no analog to previous proposals for observation of $2 \mathrm{CK}$ physics. This is in particular true for the models reviewed in Ref. [14].

The strategy of this Letter is twofold. First we perform ab initio calculations [15] to obtain an effective low-energy Hamiltonian. This is followed by a detailed analysis of the latter using analytical arguments together with a numerical analysis based on the quasiexact numerical renormalization group (NRG) [18-20].

Spin-polarized spectral densities of different orbitals are shown in Fig. 2. For the linear chain [Fig. 2(a)], we find that Co is in a $3 d^{7}$ configuration with total spin $3 / 2$. Two $3 d$ holes reside in the $x z$ and $y z$ orbitals. The remaining one is shared between the $x^{2}-y^{2}$ and $x y$ orbitals. The band dispersion (not shown) indicates that these orbitals are strongly localized, resulting in no $\mathrm{Au}$ orbitals with $x^{2}-y^{2}$ and $x y$ symmetry at the Fermi energy $\epsilon_{F}$. We obtain that the top of the $5 d x z$ and $y z$ bands of Au are slightly above $\epsilon_{F}$ [see the inset of Fig. 2(a)]. The same result was obtained by previous ab initio calculations of pure Au chains [16,21-23]. While transport experiments in $\mathrm{Au}$ chains indicate that these bands do not cross the Fermi level [24], it has been found that doping with oxygen pushes these bands up, establishing conduction through the $5 d_{x z}$ and $5 d_{y z}$ electrons of $\mathrm{Au}[25,26]$. Application of a gate voltage can have a similar effect.

The degeneracy between $x^{2}-y^{2}$ and $x y$ Co orbitals is broken in a system as represented in Fig. 1(b). Previous

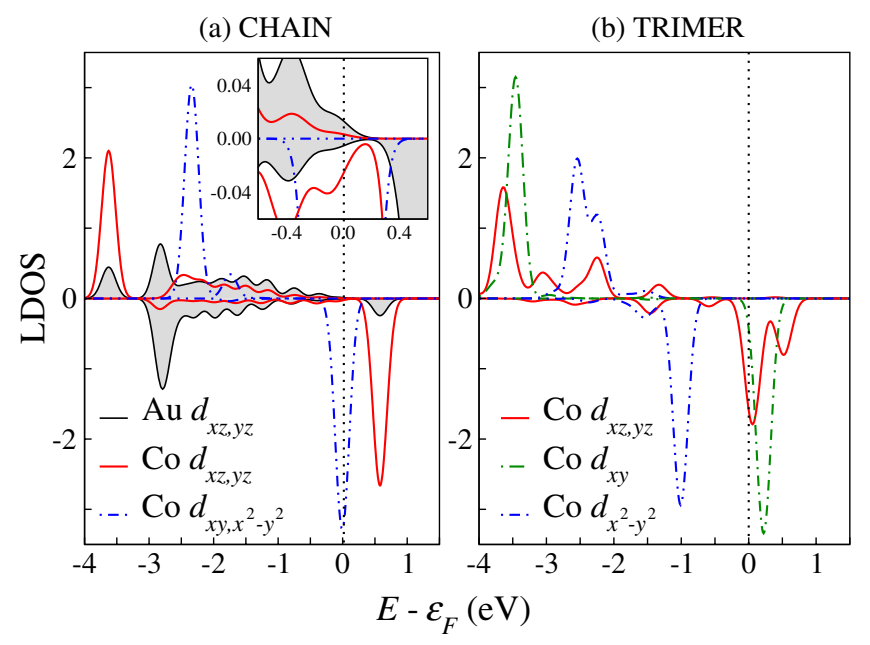

FIG. 2 (color online). Spin-polarized spectral density of different orbitals in (a) the linear chain $\mathrm{Au}_{10}$ Co depicted in Fig. 1(a) and (b) the system of Fig. 1(b). calculations for a Pt system with this geometry indicate that the $x^{2}-y^{2}$ and $x y$ orbitals are split by an energy $\Delta_{x y} \approx 1.4 \mathrm{eV}$, while the remaining orbitals are unaffected [17] (for bulk gold, one has $\Delta_{x y} \approx 1.0 \mathrm{eV}$ ). As shown in Fig. 2(b), we obtain a similar splitting here. We have taken the Au-Au and Au-Co distances as 4.93 and 4.53 a.u, respectively. Choosing $\epsilon_{F}=0$, we obtain for the average energies of the different Co $3 d$ orbitals $E_{x^{2}-y^{2}}=-1.0 \mathrm{eV}$, $E_{3 z^{2}-r^{2}}=-0.1 \mathrm{eV}$ (not shown), $E_{x y}=0.2 \mathrm{eV}$, and $E_{x z}=$ $E_{y z}=0.3 \mathrm{eV}$. The occupations indicate some admixture of the $3 d^{7}$ quadruplets with $3 d^{8}$ triplets as described below.

An important ingredient of $\hat{H}_{\text {eff }}$ is the splitting $D$ between Co spin states with projection $S_{z}= \pm 3 / 2$ from those with $S_{z}= \pm 1 / 2$ originated from spin-orbit coupling (SOC), calculated as described below.

The calculations above lead us to the following effective Anderson Hamiltonian to describe the low-energy physics,

$$
\begin{aligned}
\hat{H}_{\text {eff }} & =\sum_{M_{3}}\left(E_{3}+\frac{D}{2} M_{3}^{2}\right)\left|M_{3}\right\rangle\left\langle M_{3}\left|+E_{2} \sum_{\alpha M_{2}}\right| \alpha M_{2}\right\rangle\left\langle\alpha M_{2}\right| \\
& +\sum_{\alpha M_{2} M_{3}} \sum_{\nu k \sigma} V_{\nu}\left\langle 1 \frac{1}{2} M_{2} \sigma \mid \frac{3}{2} M_{3}\right\rangle\left(\hat{c}_{\nu k \alpha \sigma}^{\dagger}\left|\alpha M_{2}\right\rangle\left\langle M_{3}\right|+\text { H.c. }\right) \\
& +\sum_{\nu k \alpha \sigma} \epsilon_{\nu k} \hat{c}_{\nu k \alpha \sigma}^{\dagger} \hat{c}_{\nu k \alpha \sigma} .
\end{aligned}
$$

Here $E_{n}, M_{n}$ are the energies and spin projections along the chain ( $z$ direction) of the states with $n \in\{2,3\}$ holes in the $3 d$ shell of Co. The charge-transfer energy is denoted by $E_{32} \equiv E_{2}-E_{3}$. For $n=3$, the state of maximum spin projection is $|3 / 2\rangle \equiv \hat{d}_{x z \downarrow}^{\dagger} \hat{d}_{y z \downarrow}^{\dagger} \hat{d}_{x y \downarrow}^{\dagger}|0\rangle$, where $|0\rangle$ is the full $3 d^{10}$ shell and $\hat{d}_{\beta \sigma}^{\dagger}$ creates a hole with symmetry $\beta$ and spin $\sigma$. Similarly, for two holes $|\alpha 1\rangle \equiv \hat{d}_{\alpha \downarrow}|3 / 2\rangle(\alpha \in\{x z, y z\})$. The remaining relevant states at the Co site can be constructed using the spin lowering operator. The hopping amplitudes $V_{\nu}$ between the Co atom and the states $\hat{c}_{\nu k \alpha \sigma}^{\dagger}$ are assumed independent of $k$, where $\hat{c}_{\nu k \alpha \sigma}^{\dagger}$ creates a hole in the $5 d$ band of Au states with symmetry $\alpha$ at the left $(\nu=L)$ or right $(\nu=R)$ of the Co site. The hybridization of the Co atom to the mobile electrons at either side [27] is given by $\Gamma_{\nu} \equiv 2 \pi \sum_{k}\left|V_{\nu}\right|^{2} \delta\left(\omega-\epsilon_{\nu k}\right)$, neglecting the small dependence on $\omega$.

The Clebsch-Gordan coefficients $\left\langle J_{2}(1 / 2) M_{2} \sigma \mid J_{3} M_{3}\right\rangle$ lead to a spin $\mathrm{SU}(2)$ symmetric Hamiltonian for $D=0$ [28]. For any $D$, the model exhibits channel SU(2) symmetry [29] which was also exploited in the NRG calculations below [30].

Depending on the value of $D$, the model exhibits rich physics. For $D=0$, the model is an SU(2) underscreened impurity Anderson model (two channels with spin 1/2 cannot completely screen a spin $3 / 2$ ). For one channel, this model has been solved exactly [28]. In the Kondo limit $\left(E_{32} \gg \Gamma\right)$ it displays singular Fermi liquid behavior $[3,4]$ 
observed in the conductance through molecules containing $\mathrm{Co}^{+}$ions [6]. For two channels, similar physics is expected. For sufficiently negative $D,|D| \gg T_{K}$, spinflip processes are inhibited, the Co spin projection $S_{z}$ fluctuates between $3 / 2$ and 1 or between $-3 / 2$ and -1 . The properties of the system are described by two independent resonant levels [31]. For large positive $D \gg T_{K}$, we find that a Schrieffer-Wolff transformation leads to an anisotropic $2 \mathrm{CK}$ model for the interaction between an effective spin $1 / 2$ for the Co states with $S_{z}= \pm 1 / 2$ and the spin $s^{\alpha}$ of the mobile states that hybridize with the Co at the Fermi energy

$$
\hat{H}_{2 \mathrm{CK}}=J \sum_{\alpha}\left(\hat{S}_{z} \hat{S}_{z}^{\alpha}+2 \hat{S}_{x} \hat{S}_{x}^{\alpha}+2 \hat{S}_{y} \hat{S}_{y}^{\alpha}\right),
$$

where $J \simeq(2 / 3)\left(V_{L}^{2}+V_{R}^{2}\right) /\left[E_{32}-(D / 8)\right]$. The critical behavior of this model is the same as for the isotropic 2CK model [32]. As a consequence, the system displays non-Fermi liquid behavior due to overscreening of the impurity spin.

To calculate $D$ we exactly solved the $120 \times 120$ matrix of the Hamiltonian of the $3 d^{7}$ configuration, as described in Ref. [33], including the effect of SOC via $\hat{H}_{\mathrm{SOC}}=$ $\lambda \sum_{i} \hat{\mathbf{l}}_{i} \cdot \hat{\mathbf{s}}_{i}$. To gain insight on the effect of the splitting $\Delta_{x y}$ between $3 d x^{2}-y^{2}$ and $x y$ orbitals, we take the above mentioned ab initio results for the energies of the system of Fig. 1(b), but assume $E_{x y}=-E_{x^{2}-y^{2}}=\Delta_{x y} / 2$ [34]. For $\Delta_{x y}=0$, we obtain $D=-0.0426 \mathrm{eV}$, indicating that the Co spin is oriented along the chain. As discussed above, no particularly interesting physics is expected in this case. However, as $\Delta_{x y}$ increases, $D$ also increases and changes sign for $\Delta_{x y} \approx 0.8 \mathrm{eV}$. For $\Delta_{x y}=1.2 \mathrm{eV}$ [corresponding to the setup of Fig. 1(b)] we obtain $D=1.7 \mathrm{meV}$. One expects that $\Delta_{x y}$ is reduced by stretching the device and a QPT would take place if $D=0$ is reached [35].

The total resonant level width $\Gamma=\Gamma_{L}+\Gamma_{R}=0.60 \mathrm{eV}$, is determined from the width of the peak of the $x z$ and $y z$ states above the Fermi energy in the ab initio calculations. From the position of this peak, we take $E_{32}=0.3 \mathrm{eV}$, choosing the origin of one-particle energies at the Fermi energy $\boldsymbol{\epsilon}_{F}=0$. For the numerical calculations we take a band extending from $-W$ to $W$ with $W=5 \mathrm{eV}$. Repeating the $a b$ initio calculations for the system of Fig. 1(b) either without the $\mathrm{Au}$ atoms in the chain or without the left lead, we obtain $\Gamma_{R}=0.25 \mathrm{eV}, \Gamma_{L}=0.34 \mathrm{eV}$, leading to a factor $A=4 \Gamma_{L} \Gamma_{R} / \Gamma^{2}=0.977$ [36] for the conductance [see Eq. (3)].

The effective model in Eq. (1) was simulated using NRG, with the results presented in Figs. 3 and 4 [discretization parameter $\Lambda$ and truncation energy $E_{K}$ (in units of rescaled energies) are specified in the captions]. In Fig. 3 we show the impurity contribution to the entropy $S_{i}$ averaging even and odd iterations. At high temperatures, the quadruplet and both triplets are equally populated and $S_{i} \approx \ln (10)$. As the temperature is lowered below the

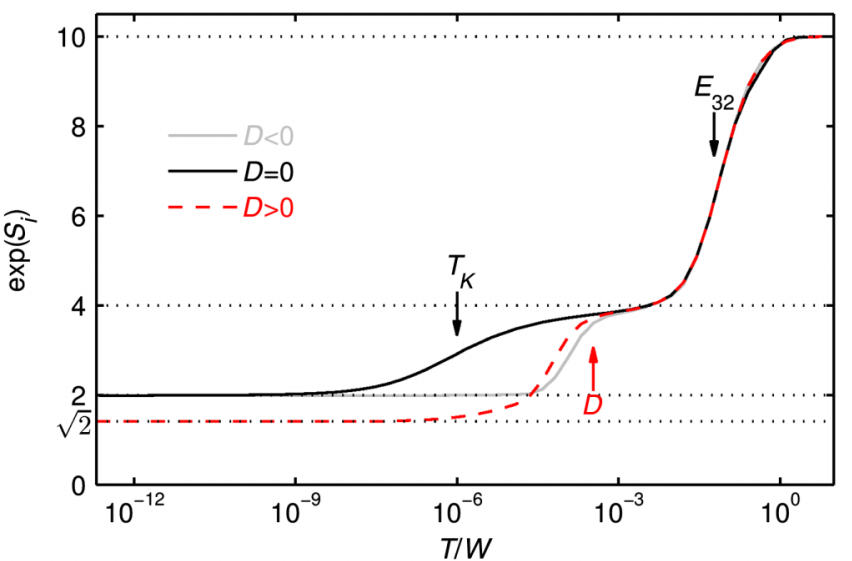

FIG. 3 (color online). Impurity contribution to the entropy as a function of temperature for $D=0$ (full line black), $D=$ $-1.7 \mathrm{meV}$ (full line gray) and $D=1.7 \mathrm{meV}$ (dashed line), calculated using $\operatorname{NRG}\left[\Lambda=3, E_{K}=6.8\right.$, keeping up to 6200 multiplets (15800 states)].

charge-transfer energy $E_{32}$, the system is in the local moment regime characterized by a local spin $3 / 2$, and therefore a plateau appears with $S_{i} \approx \ln (4)$. For $D=0$ (black solid line), as the temperature is lowered below the Kondo temperature $T_{K} / W \approx 5.2 \times 10^{-6}$, a partial screening of the local spin takes place and the ground state is a doublet with $S_{i}=\ln (2)$. The same zero-temperature value is reached for negative $D$.

In contrast, for positive $D>T_{K}, S_{i}$ starts to fall more rapidly for $T<D$, and at low temperatures it saturates at the peculiar value $\ln (2) / 2$ characteristic for $2 \mathrm{CK}$ physics. We have confirmed (not shown) that the same lowtemperature value is obtained for $D<T_{K}$. From an

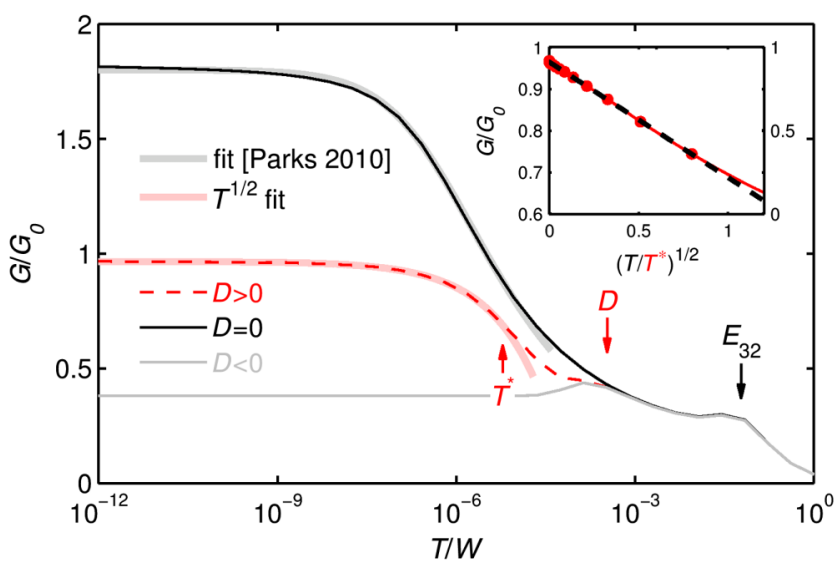

FIG. 4 (color online). Conductance as a function of temperature for $D=0$ (full black line), $D=-1.7 \mathrm{meV}$ (full gray line), and $D=1.7 \mathrm{meV}$ (dashed line), obtained with $\mathrm{NRG}[\Lambda=2$, $E_{K}=6.5$, keeping up to 12300 multiplets (31 800 states)]. The inset shows a fit of the $D>0 \mathrm{NRG}$ data with $G=a-b \sqrt{T / T^{*}}$ with $T^{*} / W=6 \times 10^{-6}$. The resulting fit is also replicated in the main panel (lower thick light line). 
analysis of the low-energy spectrum, we obtain that for $D \ll T_{K}$ the crossover occurs at an energy scale $T^{*} \ll D$, which roughly goes as $T^{*} \approx T_{K} \exp \left[-\left(6 T_{K} / D\right)^{1 / 2}\right]$. A similar crossover scale has been observed in a one-channel spin-1 UK model with anisotropy [37]. The crossover scale $T^{*}$ is present for any finite $D>0$.

Non-Fermi liquid behavior is also encountered in the spectral density of localized electrons (not shown), which at zero temperature and at the Fermi energy in the Kondo limit is half the value expected from the Friedel-Langreth sum rule [10]. These peculiarities of the spectral density manifest themselves in measurable properties, such as the total conductance through the system [27]

$$
G(T)=\frac{A e}{h} \int d \omega \pi \Gamma \rho(\omega)\left(-\frac{\partial f}{\partial \omega}\right),
$$

with $f(\omega)=1 /\left(e^{\omega / k_{B} T}+1\right)$ the Fermi function, and $\rho(\omega)=\sum_{\alpha \sigma} \rho_{\alpha \sigma}(\omega)$ the impurity spectral density of states.

The results for $G(T)$ are presented in Fig. 4. For $D=0$ the conductance at zero temperature is $G(0) \approx 1.8 G_{0}$, where $G_{0}=2 A e^{2} / h$. This is nearly the ideal value $2 G_{0}$ expected for perfect transmission with two channels. However, with increasing temperature the conductance falls more rapidly than for a usual Kondo model, reflecting the physics of the UK model [3-7]. We have obtained an excellent fit of the NRG data below $T_{K}$, using a phenomenological expression proposed for one-channel UK models [6,38] (see fit [Parks 2010] in Fig. 4).

As the anisotropy $D$ is turned on, the conductance $G(T)$ deviates from the behavior for $D=0$ for temperatures $T<|D|$. In particular, for positive $D$ the conductance at zero temperature $G(0)$ is nearly half the corresponding value for $D=0$. However, the most striking feature of $G(T)$ for $D>0$ is that it decreases with increasing temperature following a $\sqrt{T}$ behavior, instead of the characteristic $T^{2}$ dependence of a Fermi liquid (as for $D<0$ ). Fitting the results for $T<0.8 T^{*}=0.17 \mathrm{~K}$, we obtain $G / G_{0} \approx 0.96-0.47 \sqrt{T[K]}$ (see inset of Fig. 4).

The results above demonstrate measurable non-Fermiliquid properties in the model. We have checked that the results are robust and do not change if, for example, anisotropy in the triplets or more configurations of $3 d$ Co states are added. In real systems, an independent conduction channel involving $6 s$ states of Au and $4 s$ and $3 d_{3 z^{2}-r^{2}}$ states of Co contributes to the conductance, but this contribution is comparatively small because it does not lead to a resonance near the Fermi energy (in contrast to the Kondo effect) and more importantly, it does not alter the non-Fermi-liquid behavior. The latter is only affected by a magnetic field or physical ingredients that break the channel SU(2) symmetry, as a splitting $\Delta_{z}$ between Co $3 d_{x z}$ and $3 d_{y z}$ orbitals. This leads to a Fermi-liquid behavior below a temperature $T_{f}^{*} \approx\left(\nu \Delta_{z}\right)^{2} T_{K}$, where $\nu=1 / 2 W \approx 0.1 / \mathrm{eV}$ is the density of states at the Fermi energy [39]. For our parameters, $T_{K} \approx 2.6 \times 10^{-5} \mathrm{eV}$. Therefore, for moderate $\Delta_{z}, T_{f}^{*}$ is very small and non-Fermi-liquid physics is observable in a wide range of temperatures.

In summary, we have shown that it is possible to observe non-Fermi-liquid behavior in transport through a Co doped $\mathrm{Au}$ monatomic chain, in a setup that is not far from currently accessible technologies. It has the advantage over previous proposals in similar nanoscopic systems that both channels are related by symmetry and, therefore, they are truly equivalent. Moderate symmetry-breaking perturbations still allow us to observe non-Fermi-liquid physics in a wide range of temperatures. Furthermore, by stretching the system it appears feasible to induce a quantum phase transition to a phase with singular Fermi liquid behavior and increased conductance $(D=0)$ and to another phase with reduced conductance $(D<0)$.

This work was partially supported by PIP 11220080101821, PIP 00258 of CONICET, and PICT R1776 of the ANPCyT, Argentina, and also by DFG (SFB-631, WE4819/1-1; A. W.), Germany. Y. M. acknowledges funding under HGF YIG Programme VH-513. P. R. is sponsored by Escuela de Ciencia y Tecnología, Universidad Nacional de San Martín. The authors are grateful to T. Costi and A. Thiess for important discussions. S. Di Napoli is grateful to A. M. Llois and M. A. Barral for useful discussions.

[1] J. Park, A. N. Pasupathy, J. I. Goldsmith, C. Chang, Y. Yaish, J. R. Petta, M. Rinkoski, J. P. Sethna, H. D. Abruña, P. L. McEuen, and D. C. Ralph, Nature (London) 417, 722 (2002).

[2] M. Grobis, I. G. Rau, R. M. Potok, H. Shtrikman, and D. Goldhaber-Gordon, Phys. Rev. Lett. 100, 246601 (2008), and references therein.

[3] P. Mehta, N. Andrei, P. Coleman, L. Borda, and G. Zaránd, Phys. Rev. B 72, 014430 (2005).

[4] D. E. Logan, C. J. Wright, and M. R. Galpin, Phys. Rev. B 80, 125117 (2009).

[5] N. Roch, S. Florens, V. Bouchiat, W. Wernsdorfer, and F. Balestro, Nature (London) 453, 633 (2008).

[6] J. J. Parks, A. R. Champagne, T. A. Costi, W. W. Shum, A. N. Pasupathy, E. Neuscamman, S. Flores-Torres, P. S. Cornaglia, A. A. Aligia, C. A. Balseiro, G. K.-L. Chan, H. D. Abruña, and D. C. Ralph, Science 328, 1370 (2010).

[7] S. Florens, A. Freyn, N. Roch, W. Wernsdorfer, F. Balestro, P. Roura-Bas, and A. A. Aligia, J. Phys. Condens. Matter 23, 243202 (2011), and references therein.

[8] N. Andrei and C. Destri, Phys. Rev. Lett. 52, 364 (1984); A. M. Tvelick and P. B. Wiegmann, Z. Phys. B 54, 201 (1984).

[9] G. Zaránd, C-H. Chung, P. Simon, and M. Vojta, Phys. Rev. Lett. 97, 166802 (2006).

[10] A. K. Mitchell, E. Sela, and D. E. Logan, Phys. Rev. Lett. 108, 086405 (2012). 
[11] J. Bork, Y. Zhang, L. Diekhöner, L. Borda, P. Simon, J. Kroha, P. Wahl, and K. Kern, Nat. Phys. 7, 901 (2011).

[12] Y. Oreg and D. Goldhaber-Gordon, Phys. Rev. Lett. 90, 136602 (2003).

[13] R. M. Potok, I. G. Rau, H. Shtrikman, Y. Oreg, and D. Goldhaber-Gordon, Nature (London) 446, 167 (2007).

[14] D. L. Cox and A. Zawadowsky, Adv. Phys. 47, 599 (1998).

[15] We start from $a b$ initio calculations for a $\mathrm{Au}$ monatomic chain with a Co impurity [Fig. 1(a)], within the generalized gradient approximation to the density-functional theory. We employed the full-potential linearized augmented plane-wave method for one-dimensional systems, implemented in the FLEUR code, as described in Refs. [16,17]. Basis functions were expanded up to $k_{\max }=4.0 \mathrm{a.u}^{-1}$ and we have used eight $k$ points in one-half of the Brillouin zone for our self-consistent calculations. The muffin-tin radii have been set to 2.25 a.u. for all the atoms in the unit cell.

[16] Y. Mokrousov, G. Bihlmayer, and S. Blügel, Phys. Rev. B 72, 045402 (2005).

[17] A. Thiess, Y. Mokrousov, and S. Heinze, Phys. Rev. B 81, 054433 (2010).

[18] K. G. Wilson, Rev. Mod. Phys. 47, 773 (1975).

[19] R. Bulla, T. A. Costi, and T. Pruschke, Rev. Mod. Phys. 80, 395 (2008).

[20] A. Weichselbaum and J. von Delft, Phys. Rev. Lett. 99, 076402 (2007); Phys. Rev. B 86, 245124 (2012).

[21] G. Sclauzero, A. Dal Corso, and A. Smogunov, Phys. Rev. B 85, 165411 (2012).

[22] E. Yu. Zarechnaya, N. V. Skorodumova, S. I. Simak, B. Johansson, and E. I. Isaev, Comput. Mater. Sci. 43, 522 (2008).

[23] A. Delin and E. Tosatti, Phys. Rev. B 68, 144434 (2003).

[24] L. G. C. Rego, A. R. Rocha, V. Rodrigues, and D. Ugarte, Phys. Rev. B 67, 045412 (2003).

[25] S. D. Napoli, A. Thiess, S. Blügel, and Y. Mokrousov, J. Phys. Condens. Matter 24, 135501 (2012).

[26] W. H. A. Thijssen, D. Marjenburgh, R. H. Bremmer, and J. M. van Ruitenbeek, Phys. Rev. Lett. 96, 026806 (2006).

[27] Y. Meir and N. S. Wingreen, Phys. Rev. Lett. 68, 2512 (1992).
[28] A. A. Aligia, C. A. Balseiro, and C. R. Proetto, Phys. Rev. B 33, 6476 (1986).

[29] While there are four channels of mobile electrons, depending on symmetry ( $x z$ or $y z$ ) or position with respect to the Co atom ( $L$ or $R$ ), for each energy $\epsilon_{L k}=\epsilon_{R k^{\prime}}$ for which there are states at the left and the right, only the linear combination $V_{L} \hat{c}_{L k \alpha \sigma}^{\dagger}+V_{R} \hat{c}_{R k^{\prime} \alpha \sigma}^{\dagger}$ hybridizes with the Co atom. Thus, the model effectively reduces to a twochannel impurity model, with the Co atom playing the role of the impurity.

[30] A. Weichselbaum, Ann. Phys. (Amsterdam) 327, 2972 (2012).

[31] C. A. Balseiro and B. Alascio, Phys. Rev. B 26, 2615 (1982).

[32] I. Affleck, A. W. W. Ludwig, H.-B. Pang, and D. L. Cox, Phys. Rev. B 45, 7918 (1992).

[33] A. A. Aligia and T. Kroll, Phys. Rev. B 81, 195113 (2010).

[34] The calculation also requires the Coulomb integrals $F_{i}$ [33] and the SOC strength $\lambda$. From a fit of the low energy spectra of late transition metal atoms, we take $F_{2}=$ $0.16 \mathrm{eV}, F_{4}=0.011 \mathrm{eV}$, and $\lambda=0.08 \mathrm{eV}$.

[35] We find from our numerics that the anisotropy in Eq. (1), which solely acts on the states $\left|M_{3}\right\rangle$, can be generalized to a uniform anisotropy $(D / 2)\left(S_{z \text {,imp }}^{\text {tot }}\right)^{2}$ without much affecting the overall physics. Hence, for simplicity, a uniform anisotropy $D$ has been used.

[36] The total hybridization $\Gamma \equiv \Gamma_{L}+\Gamma_{R}=0.59 \mathrm{eV}$, obtained by fitting the widths of the peaks when either the part of the system to the right or to the left of the Co atom is cut off, does not exactly coincide with the total $\Gamma=$ $0.60 \mathrm{eV}$ obtained for the whole system. However, it is very close. We used $\Gamma$ in the NRG calculations and $\Gamma_{L}$ and $\Gamma_{R}$ to calculate the asymmetry factor $A=$ $4 \Gamma_{L} \Gamma_{R} /\left(\Gamma_{L}+\Gamma_{R}\right)^{2}$.

[37] P. S. Cornaglia, P. Roura-Bas, A. A. Aligia, and C. A. Balseiro, Europhys. Lett. 93, 47005 (2011).

[38] The fit [Parks 2010] for $D=0$ refers to Eqs. (S1)-(S3) of Ref. [6], with resulting parameters $\xi=0.69, \alpha=0.358$, $\Delta=0.022$, and $T_{K}=5.2 \times 10^{-6} \mathrm{~W}=0.30 \mathrm{~K}$.

[39] E. Sela, A. K. Mitchell, and L. Fritz, Phys. Rev. Lett. 106, 147202 (2011). 